\title{
Aplicación de quitosano incrementa la emergencia, crecimiento y rendimiento del cutivo de tomate (Solanum lycopersicum L.) en condiciones de invernadero
}

\author{
Chitosan application increases the emergence, growth and yield of tomato crop \\ (Solanum lycopersicum L.) under greenhouse conditions
}

\begin{abstract}
Juan José Reyes-Pérez ${ }^{1}$, Marisol Rivero-Herrada', Erick Leonardo García-Bustamante ${ }^{1}$, Félix Alfredo Beltran-Morales² y Francisco Higinio Ruiz-Espinoza ${ }^{2 *}$

Universidad Técnica Estatal de Quevedo. Av. Quito. Km 1 1⁄2 vía a Santo Domingo. Quevedo, Los Ríos, Ecuador.

Universidad Autónoma de Baja California Sur, Carretera al Sur Km 5.5. La Paz, Baja California Sur, México. AP-19B.
\end{abstract}

\section{RESUMEN}

La agricultura actual busca productos que permitan el crecimiento y desarrollo de los cultivos, así como incrementar los rendimientos, asimismo que los principios activos sean de origen natural, biodegradables y no causen daños al ambiente. El propósito de esta investigación fue evaluar el efecto de la aplicación de quitosano en dosis de 1, 2 y $3 \mathrm{~g} \mathrm{~L}^{-1}$ en la emergencia, desarrollo y rendimiento de las variedades de tomate Floradade y Amalia, en condiciones de invernadero. El diseño experimental fue un Bloques completos al azar, con arreglo factorial $2 \times 3+2$. Los resultados mostraron que la dosis de $2 \mathrm{~g} \mathrm{~L}^{-1}$ fue la que mostró los valores mayores en la emergencia y desarrollo de las plantas de tomate, estimuló una mayor fructificación, peso de frutos y mayor rendimiento en la variedad Floradade. A esto se concluye que la aplicación de quitosano en dosis de $2 \mathrm{~g} \mathrm{~L}^{-1}$ en las condiciones que se realizó la investigación incrementó los rendimientos del cultivo de tomate en la variedad Floradade.

Palabras clave: Dosis, Floradade, Amalia, variables morfométricas, rendimiento.

\section{ABSTRACT}

Current agriculture looks for products that allow the growth and development of crops, as well as increase yields, and also that the active principles are of natural origin, biodegradable and do not cause damage to the environment. The purpose of this research was to evaluate the effect of the application of chitosan at doses of 1, 2 and $3 \mathrm{~g} \mathrm{~L}^{-1}$ in the emergence, development and performance of the Floradade and Amalia tomato varieties, under greenhouse conditions. The experimental design was a randomized complete block, with a $2 \times 3$ +2 factorial arrangement. The results showed that the $2 \mathrm{~g} \mathrm{~L}^{-1}$ dose was the one with the highest values in the emergence and development of tomato plants, stimulating higher fruiting, fruit weight and higher yield in the Floradade variety. It is concluded that the application of chitosan at doses of $2 \mathrm{~g}$ ${ }^{\mathrm{L}-1}$ under our experimental conditions, increased the yields of the tomato crop in the Floradade variety.

Key words: Dose, Floradade, Amalia, morphometric variables, yield.

\section{INTRODUCCIÓN}

El cultivo de tomate (Solanum lycopersicum L.) es de gran importancia en el mundo por el volumen de producción, como por su aceptación en fresco, salsas, sopas, procesado, entre otras (Reyes et al., 2020). Dado al estatus que mantiene de ser la hortaliza más consumida y de mayor valor económico, es cultivada en diferentes países, entre los cuales se destacan China, India, Estados Unidos, Turquía, Marruecos, México y Egipto (Cestoni et al., 2006). La producción mundial de tomate está en constante crecimiento, no solo por el aumento de las áreas cultivadas, sino también porque los agricultores aplican tecnologías que les permiten elevar los rendimientos (Feicán et al., 2016).

La producción de tomate en el Ecuador se ha incrementado considerablemente, especialmente en las zonas de la región Serrana, donde destacan Chimborazo, y en la Costa Santa Elena (Coronel, 2009). Según cifras del Sistema de Información Pública Agropecuaria (SIPA, 2018), se cultivan alrededor de 1970 hectáreas de tomate, distribuidas en la regiones Costa y Sierra, con una producción de 62, 675 toneladas y un promedio de rendimiento de $32.07 \mathrm{t} \mathrm{ha}^{-1}$ en campo abierto y 250 t ha-1 $^{-1}$ en invernadero. Betancourt (2014) quien afirma que comúnmente en la serranía de Ecuador se produce el tomate riñón de mesa y en el litoral el tomate industrial.

Actualmente, para la producción del cultivo de tomate se recurre al uso excesivo de fertilizantes, además de pesticidas para el control de plagas y enfermedades. Las altas dosis de pesticidas y fertilizantes son factores que provocan efectos sobre el $\mathrm{pH}$ del suelo, desbalance nutricional, pérdida de microorganismos benéficos entre otros (Múnera, 2015).

Además pese a que con el uso de pesticidas, la producción de alimentos ha aumentado en un 50\%, desafortunadamente esto a forjando riesgos que se manifiestan en nuevas patologías, resultantes de la exposición a estos tóxicos (Devine et al., 2008). Para la producción de este cultivo se necesitan cada vez dosis mayores de fertilizantes, además de verse limitada severamente por problemas fitosanitarios, entre las causas de la disminución del rendimiento se atribuye al incremento de la incidencia y severidad de enfermedades, insectos plagas y de nemátodos agalladores (MAGAP, 2014).

*Autor para correspondencia: Francisco Higinio Ruiz Espinoza Correo electrónico: fruiz@uabcs.mx

Recibido: 28 de julio de 2020 Aceptado: 13 de agosto de 2020 
Los bioestimulantes son sustancias y/o microorganismos que promueven el crecimiento y desarrollo de las plantas, además de mejorar la fotosíntesis (Morocho y Leiva, 2019). Independientemente de su contenido hormonas vegetales, enzimas vitaminas entre otros, pueden contener sustancias, compuestos, y/o microorganismos, cuyo uso funcional, cuando se aplican a las plantas o la rizosfera, ya sea vía foliar o en el suelo, implica la mejoría del desarrollo del cultivo, vigor, rendimiento y/o la calidad la planta mediante la estimulación del crecimiento y las respuestas a estrés biótico y/o abiótico (Brown y Saa, 2015).

El quitosano es un polisacárido natural que ha sido utilizado frecuentemente en el desarrollo de distintos materiales, debido a sus excepcionales propiedades fisicoquímicas y biológicas (Caro et al., 2019). Es un producto que se obtiene principalmente de subproductos de la industria pesquera, en especial del exosqueleto de los crustáceos, el cual es copolímero lineal formado por unidades de glucosamina y en menor medida de $\mathrm{N}$-acetil D-glucosamina unidos por enlaces $\beta$ 1-4 y además ha demostrado tener una actividad bioestimulante en el crecimiento y rendimiento de las hortalizas (Ramírez et al., 2010). Aunque siempre se ha asociado su uso con la agricultura ecológica, está cobrando cada vez más protagonismo en la agricultura tradicional (Rodríguez et al., 2009). Es un producto compatible con tejidos de plantas y animales. Su aplicación en la agricultura, es muy importante por la promoción del crecimiento de las plantas e inhibición del desarrollo de fitopatógenos (González et al., 2017; Rodríguez et al., 2015; Berumen, 2015).

Los bioestimulantes con quitosano puede mejorar la asimilación de los nutrientes en cultivos hortícolas de interés agroeconómico como el tomate, además de ser una potencial alternativa para reducir la incidencia por el ataque de fitopatógenos logrando producciones sostenibles y económicamente justificables (Falcón et al., 2015).

El quitosano podría representar una alternativa viable para aumentar los rendimientos, reducir la contaminación ambiental por el excesivo uso de fertilizantes y pesticidas además de producir alimentos más inocuos con menos riesgo para el consumo humano, a esto se suma la ventaja de reducir los costos de producción y de esta forma mejorar la calidad de vida de los agricultores (Reyes et al., 2019; Torres et al., 2018).

Por lo anterior, el objetivo de la investigación fue evaluar el efecto del quitosano en el crecimiento y desarrollo de variedades de tomate en condiciones de invernadero.

\section{MATERIALES Y MÉTODOS \\ Descripción del área de estudio}

La investigación se llevó a cabo en la finca Experimental "La María", propiedad de la Universidad Técnica Estatal de Quevedo, ubicada en el Km 7 Vía Quevedo-El Empalme, cuyas coordenadas geográficas son: $79^{\circ} 27^{\prime}$ de longitud Oeste y $01^{\circ} 06^{\prime}$ de latitud Sur a una altitud de $120 \mathrm{msnm}$ con una duración del experimento de 120 días. Esta se llevó a cabo en condiciones de invernadero. El cultivo estudiado fue el tomate (Solanum lycopersicum L.), cultivares "Floradade y Amalia. Las semillas se desinfectaron y se trataron con $10 \mathrm{~mL}$ de hipoclorito de calcio al 5\%, durante cuatro horas.

Las semillas de tomate desinfectadas se colocaron en placas Petri con papel de filtro humedecido durante $72 \mathrm{~h}$ en oscuridad. Posteriormente, se sembraron en cepellones que contenían como sustrato suelo, abono orgánico (composta) y arena (esterilizada) en proporción 4:2:1. Después de 20 días de sembradas las plántulas de tomate, de alrededor de 15 $\mathrm{cm}$ de altura, se realizó el trasplante en macetas de $12 \mathrm{~L}$ en una densidad de plantación de 4 plantas por metro lineal, las macetas se mantuvieron dentro de un invernadero, el riego se realizó cada tercer día, la fertilización solo fueron las diferentes concentraciones de quitosano $\left(1,2\right.$ y $3 \mathrm{~g} \mathrm{~L}^{-1}$ preparadas con agua destilada) se aplicó $0.1 \mathrm{~mL}$ por plántula, como control se utilizó agua destilada, todos los tratamientos se aplicaron vía foliar, asperjados con un atomizador.

\section{Diseño experimental}

Se evaluaron 8 tratamientos (Tabla 1), consistentes en la aplicación de quitosano en 1, 2 y $3 \mathrm{~g} \mathrm{~L}^{-1}$ y dos variedades de tomate (Floradade y Amalia) y un control de cada variedad con agua destilada, utilizando un diseño experimental de bloques completos al azar con arreglo factorial $2 \times 3+2$ aplicando tres réplicas por tratamiento de manera aleatoria. Cada bandeja de 200 cavidades se consideró una repetición.

Tabla 1. Tratamientos evaluados en la investigación.

Table 1. Treatments evaluated in the research.

\begin{tabular}{lccc}
\hline Tratamientos & Variedad & Dosis L $^{-1}$ & $\begin{array}{c}\text { Nomenclatura } \\
\text { tratamientos }\end{array}$ \\
\hline T1 & Floradade & 1 & $\mathrm{~V}_{1} \mathrm{~B}_{1}$ \\
T2 & Floradade & 2 & $\mathrm{~V}_{1} \mathrm{~B}_{2}$ \\
T3 & Floradade & 3 & $\mathrm{~V}_{1} \mathrm{~B}_{3}$ \\
T4 & Amalia & 1 & $\mathrm{~V}_{2} \mathrm{~B}_{1}$ \\
T5 & Amalia & 2 & $\mathrm{~V}_{2} \mathrm{~B}_{2}$ \\
T6 & Amalia & 3 & $\mathrm{~V}_{3} \mathrm{~B}_{3}$ \\
T7 & Floradade & Agua destilada & Control \\
T8 & Amalia & Agua destilada & Control \\
\hline
\end{tabular}

Las variables fisiológicas de semillas evaluadas fueron porcentaje de emergencia, esta se registró diariamente y el porcentaje final se determinó a los catorce días, se calculó mediante la ecuación de Maguire (1962):; donde $\mathrm{n}_{1}, \mathrm{n}_{2^{\prime}} \ldots \mathrm{n}_{20}$ son el número de semillas germinadas en los tiempos $\mathrm{t}_{1}, \mathrm{t}_{2^{\prime}}$ ... $\mathrm{t}_{14}$ (en días), el porcentaje de germinación, , a) variables morfométricas, como longitud de tallo, longitud de radícula, biomasa fresca y seca de parte aérea (tallos + hojas) y de radícula, b) indicadores de crecimiento de las plantas a los 45 días después de trasplante (ddt): altura de la planta $(\mathrm{cm})$, diámetro del tallo $(\mathrm{cm}), \mathrm{c}$ ) indicadores de crecimiento de los frutos a los $65 \mathrm{ddt}$ : masa fresca del fruto (g), diámetro polar $(\mathrm{cm})$ : Por cada tratamiento se seleccionaron 40 frutos y se midió con un calibrador de vernier, diámetro ecuatorial $(\mathrm{cm})$; por cada tratamiento se seleccionaron 40 frutos y se midió 
con un calibrador de vernier d) indicadores de la producción: Número de fruto por planta (se utilizó el valor promedio de los frutos obtenidos a partir de 10 plantas) y Rendimiento agrícola ( $\mathrm{kg} \mathrm{ha}^{-1}$ ): la producción agrícola del cultivo en cada cosecha se determinó por peso de los frutos en cada parcela.

\section{Procesamiento estadístico}

Los datos se procesaron a través del paquete estadístico Statistica v. 10.0 para Windows empleando un análisis de varianza de clasificación simple. En los casos en que los indicadores mostraron diferencias estadísticas significativas, se utilizó la prueba de comparación múltiple de medias de Tukey $(\mathrm{P} \leq 0,05)$.

\section{RESULTADOS Y DISCUSIÓN}

Al analizar las variables fisiológicas de semillas de tomate en las variedades Floradade y Amalia con aplicación de quitosano, se obtuvo que la aplicación de quitosano en dosis de $2 \mathrm{~g} \mathrm{~L}^{-1}$ propició un mayor número de semillas germinadas, con un porcentaje de $15 \%$ mayor que en los otros tratamientos, mientras las dosis 1 y $3 \mathrm{~g} \mathrm{~L}^{-1}$ no presentaron diferencias estadísticas en la germinación (Figura 1A). En cuanto a la capacidad de emergencia, las dosis de $2 \mathrm{~g} \mathrm{~L}^{-1}$ en la variedad Amalia y Floradade presentaron los valores de porcentaje mayores en $12.5 \%$ de emergencia a los controles, mientras que las dosis de 1 y $3 \mathrm{~g} \mathrm{~L}^{-1}$ no tuvieron ningún efecto en el porcentaje de emergencia (Figura 1B). En lo que respecta a la tasa de emergencia, las dosis de quitosano de 1 y $2 \mathrm{~g} \mathrm{~L}^{-1}$ en la variedad Floradade y la dosis de $2 \mathrm{~g} \mathrm{~L}^{-1}$ en la variedad Amalia, incrementaron la tasa de emergencia de las semillas evaluadas, por su parte la dosis de $3 \mathrm{~g} \mathrm{~L}^{-1}$ y el control no tuvieron ningún efecto en la tasa de emergencia (Figura 1C).

Los resultados obtenidos sobre el efecto del quitosano en la emergencia de semillas de coincide con lo reportado por Gonzales et al. (2017). quienes al evaluar la respuesta agronómica del cultivo del tomate (Solanum lycopersicum, L) a la aplicación de quitosano manifiesta que se obtuvieron resultados cuando las semillas fueron embebidas con una solución de $300 \mathrm{mg}$. Por otra parte Velásquez (2008) menciona que la aplicación de quitosano ha mostrado efectos positivos en la estimulación de la emergencia de las semillas como el crecimiento de partes de la planta tales raíces, retoños y hojas. En este sentido Lee et al. (2005) establece que, las mismas están involucradas en procesos fisiológicos, ya que contribuyen a evitar las pérdidas de agua por vía de la transpiración, aspecto de gran importancia para este cultivo en particular, por la gran demanda de este elemento para realizar sus diferentes funciones.

En lo que respecta a las variables de crecimiento se observó que, la imbibición de las semillas de tomate en soluciones de quitosano ejerció un efecto positivo en la longitud de las raíces $(P \leq 0.05)$. Con las concentraciones de 1,0 y $2,0 \mathrm{~g}$
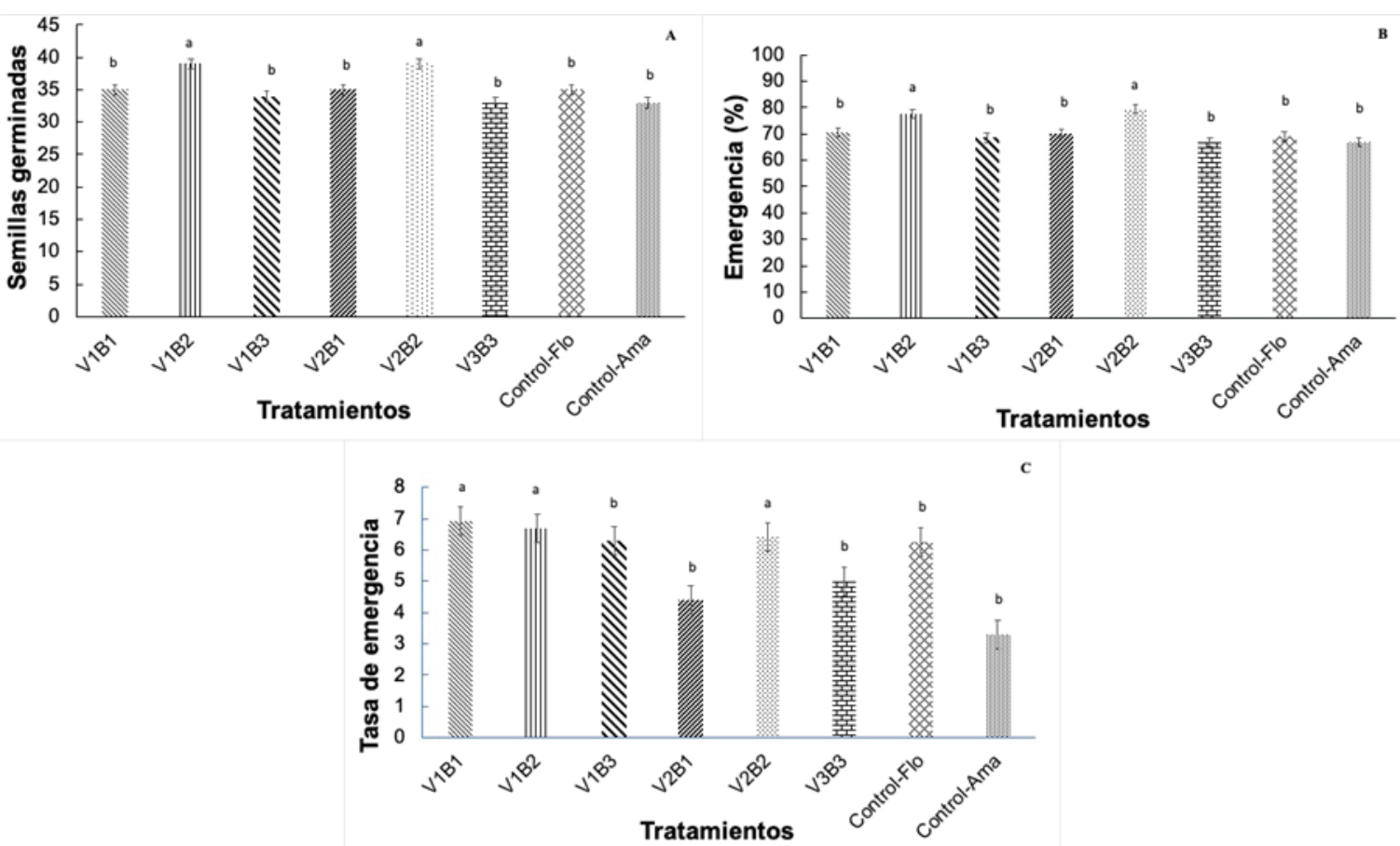

Figura 1. Número de semillas germinadas (A), emergencia (B) y tasa de emergencia (C) de las variedades Floradade y Amalia mediante la aplicación de tres dosis de quitosano. Las barras en las columnas indican error estándar ( $\pm \mathrm{ES})$; diferentes letras indican diferencias significativas entre los tratamientos (Tukey $\mathrm{p} \leq 0.05$ ). $\mathrm{V}_{1} \mathrm{~B}_{1}=$ Floradade $1 \mathrm{~g} \mathrm{~L}^{-1}, \mathrm{~V}_{1} \mathrm{~B}_{2}=$ Floradade $2 \mathrm{~g} \mathrm{~L}^{-1}, \mathrm{~V}_{1} \mathrm{~B}_{3}=$ Floradade $3 \mathrm{~g}$ $\mathrm{L}^{-1}, \mathrm{~V}_{2} \mathrm{~B}_{1}=$ Amalia $1 \mathrm{~g} \mathrm{~L}^{-1}, \mathrm{~V}_{2} \mathrm{~B}_{2}=$ Amalia $2 \mathrm{~g} \mathrm{~L}^{-1}, \mathrm{~V}_{3} \mathrm{~B}_{3}=$ Amalia $3 \mathrm{~g} \mathrm{~L}^{-1}$, control-flo (Floradade agua destilada), control-ama (Amalia agua destilada).

Figure 1. Number of germinated seeds (A), emergence (B) and emergence rate (C) of the Floradade and Amalia varieties, through the application of three doses of chitosan. Error bars indicate $\pm \mathrm{ES}$; different letters indicate significant differences between treatments (Tukey $\mathrm{p} \leq 0.05$ ). $\mathrm{V}_{1} \mathrm{~B}_{1}=$ Floradade $1 \mathrm{~g} \mathrm{~L}^{-1}, \mathrm{~V}_{1} \mathrm{~B}_{2}=$ Floradade $2 \mathrm{~g} \mathrm{~L}^{-1}, \mathrm{~V}_{1} \mathrm{~B}_{3}=$ Floradade $3 \mathrm{~g} \mathrm{~L}^{-1}, \mathrm{~V}_{2} \mathrm{~B}_{1}=$ Amalia $1 \mathrm{~g} \mathrm{~L}^{-1}, \mathrm{~V}_{2} \mathrm{~B}_{2}=\mathrm{Amalia}_{2} \mathrm{~g} \mathrm{~L}^{-1}$, $\mathrm{V}_{3} \mathrm{~B}_{3}=$ Amalia $3 \mathrm{~g} \mathrm{~L}^{-1}$, control-flo (Floradade distilled water), control-ama (Amalia distilled water). 
$\mathrm{L}^{-1}$, se alcanzaron valores superiores en la variedad Floradade, sin embargo no se presentó diferencias con el control en ambas variedades (Figura $2 \mathrm{~A}$ ).

Mientras tanto la variable diámetro del tallo a los 15 días de evaluación mostró que las dosis de 1 y $2 \mathrm{~g} \mathrm{~L}^{-1}$ en la variedad Floradade y el control en ambas variedades presentaron los más altos promedios, pero sin diferencia entre la dosis de 2 y $3 \mathrm{~g} \mathrm{~L}^{-1}$ en la variedad Floradade y 1,2 y $3 \mathrm{~g} \mathrm{~L}^{-1} \mathrm{en} \mathrm{la}$ variedad Amalia (Figura 2B).

En lo que respecta a los 45 días de evaluación, no existieron diferencias significativas entre los tratamientos evaluados en la variable diámetro del tallo.

En lo referente a la altura de planta a los 15 días después de la siembra (Figura 2C), dio como resultado que la variedad Amalia a la aplicación de las dosis 2 y $3 \mathrm{~g} \mathrm{~L}^{-1}$ reprimió inicialmente el crecimiento. Sin embargo no existieron diferencias significativas entre el control con aplicación de agua destilada y la dosis de quitosano de $1 \mathrm{~g} \mathrm{~L}^{-1}$. Por su parte la variedad Floradade a los 15 días después de siembra (dds), el control mostró el mayor valor, mientras que las dosis 1 y $3 \mathrm{~g}$ $\mathrm{L}^{-1}$ no mostraron diferencias, mientras que en la dosis de $2 \mathrm{~g}$ $\mathrm{L}^{-1}$ el crecimiento fue inhibido.
Por su parte a los 45 días de evaluación, la aplicación de 1 y $2 \mathrm{~g} \mathrm{~L}^{-1}$ estimuló un mayor crecimiento al que existió inicialmente a los 15 días de evaluación por la aplicación de las dosis más altas en ambas variedades, pero sin diferencias con el control en ambas variedades. La dosis mayor $\left(3 \mathrm{~g} \mathrm{~L}^{-1}\right)$ presentó valores menores en ambas variedades.

La variable de biomasa fresca (Figura 2D) de las plantas sometidas a los tratamientos 15 días después de la siembra el análisis mostró que, la aplicación de la dosis de quitosano de $2 \mathrm{~g} \mathrm{~L}^{-1}$, presentó el mayor promedio de biomasa fresca de planta, sin embargo este no difirió de la dosis de $1 \mathrm{~g} \mathrm{~L}^{-1}$, mientras la biomasa fresca de menor promedio se presentó cuando se aplicó una dosis de $3 \mathrm{~g} \mathrm{~L}^{-1}$.

La aplicación de $2 \mathrm{~g} \mathrm{~L}^{-1}$ en la variedad Floradade presentó la mayor biomasa fresca de planta, sin embargo estadísticamente no presentó diferencias con los demás tratamientos evaluados $(P \leq 0.05)$.

El quitosano ha sido reconocido como un bioestimulante del crecimiento vegetal (González et al., 2014; Pichyangkuraa y Chadchawanb, 2015). Su mecanismo de acción se ha relacionado con un incremento del contenido de clorofila en las hojas de la planta luego de la aplicación foliar de este
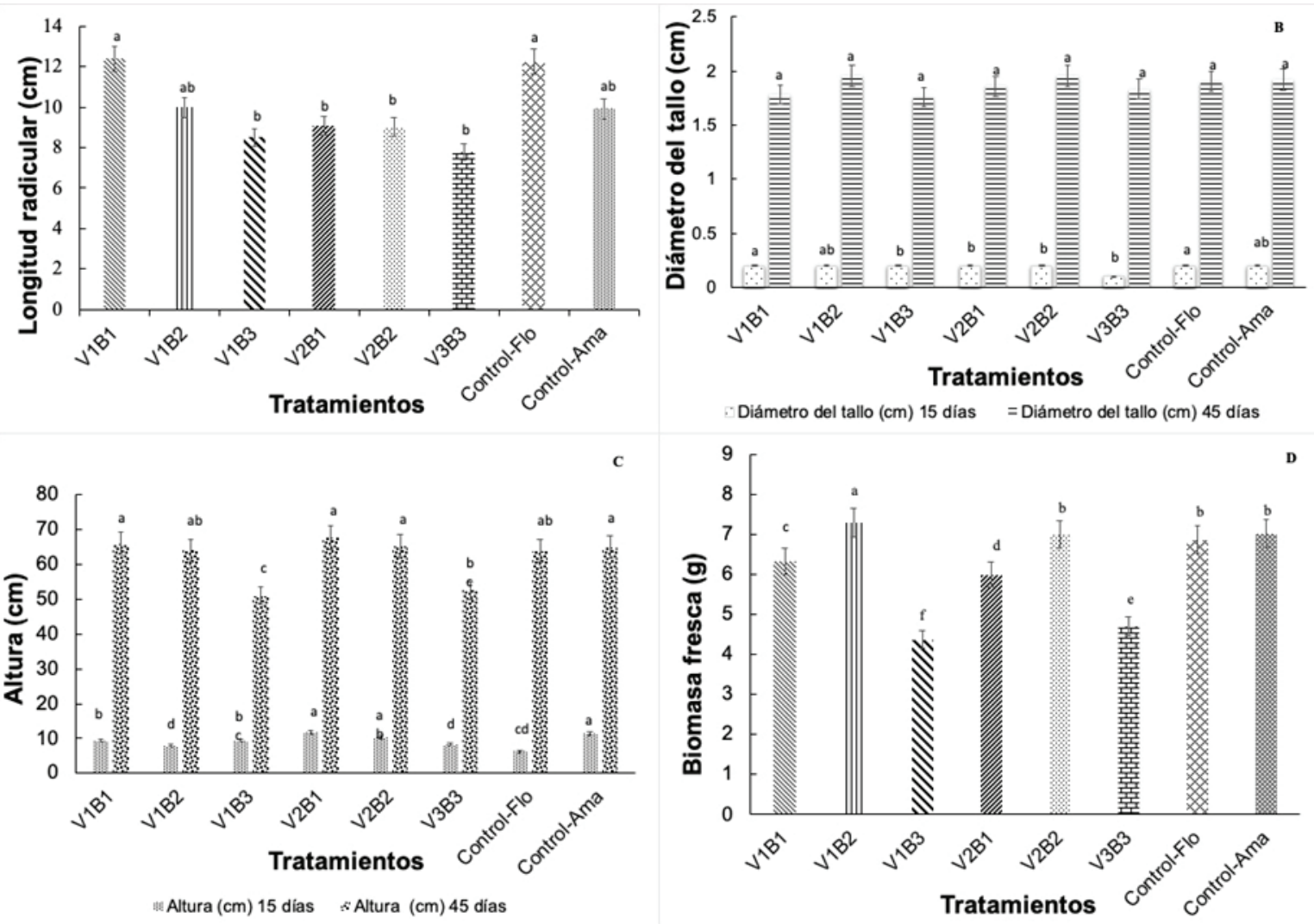

Figura 2. Longitud radicular (A), diámetro del tallo (B) altura (C) y biomasa fresca (D) de las variedades Floradade y Amalia mediante la aplicación de tres dosis de quitosano. Las barras en las columnas indican error estándar ( $\pm E S$ ); diferentes letras indican diferencias significativas entre los tratamientos (Tukey $\mathrm{p} \leq 0.05$ ). $\mathrm{V}_{1} \mathrm{~B}_{1}=$ Floradade $1 \mathrm{~g} \mathrm{~L}^{-1}, \mathrm{~V}_{1} \mathrm{~B}_{2}=$ Floradade $2 \mathrm{~g} \mathrm{~L}^{-1}, \mathrm{~V}_{1} \mathrm{~B}_{3}=$ Floradade $3 \mathrm{~g} \mathrm{~L}^{-1}, \mathrm{~V}_{2} \mathrm{~B}_{1}=$ Amalia $1 \mathrm{~g} \mathrm{~L}^{-1}, \mathrm{~V}_{2} \mathrm{~B}_{2}=$ Amalia $2 \mathrm{~g} \mathrm{~L}^{-1}, \mathrm{~V}_{3} \mathrm{~B}_{3}=$ Amalia $3 \mathrm{~g} \mathrm{~L}^{-1}$, control-flo (Floradade agua destilada), control-ama (Amalia agua destilada). Figure 2. Root length (A), stem diameter (B) height (C) and fresh biomass (D) of the Floradade and Amalia varieties, through the application of three doses of chitosan. Error bars indicate $\pm \mathrm{ES}$; different letters indicate significant differences between treatments (Tukey $\mathrm{p} \leq 0.05$ ). $\mathrm{V}_{1} \mathrm{~B}_{1}=$ Floradade $1 \mathrm{~g} \mathrm{~L}^{-1}, \mathrm{~V}_{1} \mathrm{~B}_{2}=$ Floradade $2 \mathrm{~g} \mathrm{~L}^{-1}, \mathrm{~V}_{1} \mathrm{~B}_{3}=$ Floradade $3 \mathrm{~g} \mathrm{~L}^{-1}, \mathrm{~V}_{2} \mathrm{~B}_{1}=$ Amalia $1 \mathrm{~g} \mathrm{~L}^{-1}, \mathrm{~V}_{2} \mathrm{~B}_{2}=\mathrm{Amalia}_{2} \mathrm{~g} \mathrm{~L}^{-1}$, $\mathrm{V}_{3} \mathrm{~B}_{3}=$ Amalia $3 \mathrm{~g} \mathrm{~L}^{-1}$, control-flo (Floradade distilled water), control-ama (Amalia distilled water). 
polímero. Condición que puede varian de acuerdo a la concentración de quitosano utilizada (Salachna y Zawadzinska, 2014). Por su parte Rendina et al. (2019), establece que al aplicar quitosano, este presento efectos positivos en pepino (Cucumis sativus) en las variables de crecimiento y desarrollo el cual estimula un incremento de la producción de clorofila y la fotosíntesis en las plantas, así como el uso más eficiente del agua mediante un cierre estomático (Iriti y Faoro, 2009), tal como los resultados obtenidos en nuestra investigación al aplicar la dosis de $2 \mathrm{~g} \mathrm{~L}^{-1}$ esto pudiera deberse que los productos de la descomposición de la quitina en el suelo tales como amonio y sus derivados, que pueden constituirse en una fuente lenta pero sostenida de nitrógeno, cuya duración puede llegar incluso a los tres meses (Beier y Bertilsson, 2013), por lo que en estas circunstancias promueven el desarrollo de plantas más saludables y menos susceptibles al ataque de patógenos (Kumeta et al., 2018). En este mismo sentido otros autores (Terry et al., 2017; Rodríguez et al., 2017; Reyes et al., 2020), reportan resultados similares en la altura de cultivos como tomate y arroz (Oriza sativa). De la misma manera González et al. (2014), al evaluar quitosano en el crecimiento en tomate (Solanum lycopersicum L.) encontraron resultados similares a los de esta investigación, sin embargo la dosis de mayor respuesta en dicha investigación fue de $0,1 \mathrm{~g} \mathrm{~L}^{-1}$. Por su parte Terry et al. (2017) al evaluar quitomax ${ }^{\circledast}$ en tomate encontraron que los mejores promedios de altura de planta, longitud radicular y diámetro de tallo se observaron con la aplicación de $1 \mathrm{~g} \mathrm{~L}^{-1}$ lo que no coincide con los resultados de nuestra esta investigación, donde el mejor tratamiento en ambas variedades fue de $2.0 \mathrm{~g} \mathrm{~L}^{-1}$.

La aplicación de quitosano mejoró notoriamente el diámetro de frutos en ambas variedades evaluadas (Figura 3A). La aplicación de $2 \mathrm{~g} \mathrm{~L}^{-1}$ en la variedad Floradade estimuló al desrrollo de frutos de mayor tamaño con un diámetro de frutos entre 4.93-5.07 cm. El menor diámetro se registró en los frutos del tratamiento control.

En la variedad Amalia se presentó el menor diámetro ecuatorial, sin embargo se observó que la aplicación de quitosano incrementa el tamaño del fruto destacándose la dosis de $2 \mathrm{~g} \mathrm{~L}^{-1}$.

En la misma Figura 3B se observa lo referente al diámetro polar, donde la aplicación de quitosano en cada una de las variedades. La dosis de $2 \mathrm{~g} \mathrm{~L}^{-1}$ en la variedad Floradade estimuló una producción de frutos de mayor tamaño con un promedio de $4.76 \mathrm{~cm}$. El menor diámetro se registró con la aplicación de agua. Por su parte la variedad Amalia presentó el menor diámetro polar, sin embargo se observó que la aplicación de quitosano incrementa el tamaño del fruto destacándose la aplicación de 2 y $3 \mathrm{~g} \mathrm{~L}^{-1}$ con promedios de 4.23 y $4.20 \mathrm{~g}$ respectivamente superando al control.

El tratamiento conformado por la aplicación de quitosano en dosis de $2 \mathrm{~g} \mathrm{~L}^{-1}$ en la variedad floradade estimuló una mayor producción de frutos por planta (Figura 4A), pero estadísticamente $(P \leq 0.05)$ igual a las dosis de $1 \mathrm{~g} \mathrm{~L}^{-1}$ para esta misma variedad y las dosis de 2 y $3 \mathrm{~g} \mathrm{~L}^{-1}$ de la variedad Amalia. Mientras que la dosis de quitosano $3 \mathrm{~g} \mathrm{~L}^{-1}$ redujo significativamente el promedio de frutos en la variedad Floradade.

En la Figura 4B, se puede observar el peso del fruto, donde el análisis de varianza presentó diferencias significativas $(P \leq 0.05)$ entre los tratamientos, la dosis de $2 \mathrm{~g} \mathrm{~L}^{-1}$ estimuló un mayor peso de fruto en la variedad Floradade, seguido de la aplicación de quitosano a razón de $3 \mathrm{~g} \mathrm{~L}^{-1}$ de esta misma variedad. Por su parte la variedad Amalia presentó el valor menor en todos los tratamientos.

Por su parte el rendimiento (Figura 4C) con aplicación de quitosano estimuló un incremento del rendimiento con respecto a los controles evaluados (agua destilada en ambas variedades). La dosis de $2 \mathrm{~g} \mathrm{~L}^{-1}$ estimuló un incremento en el
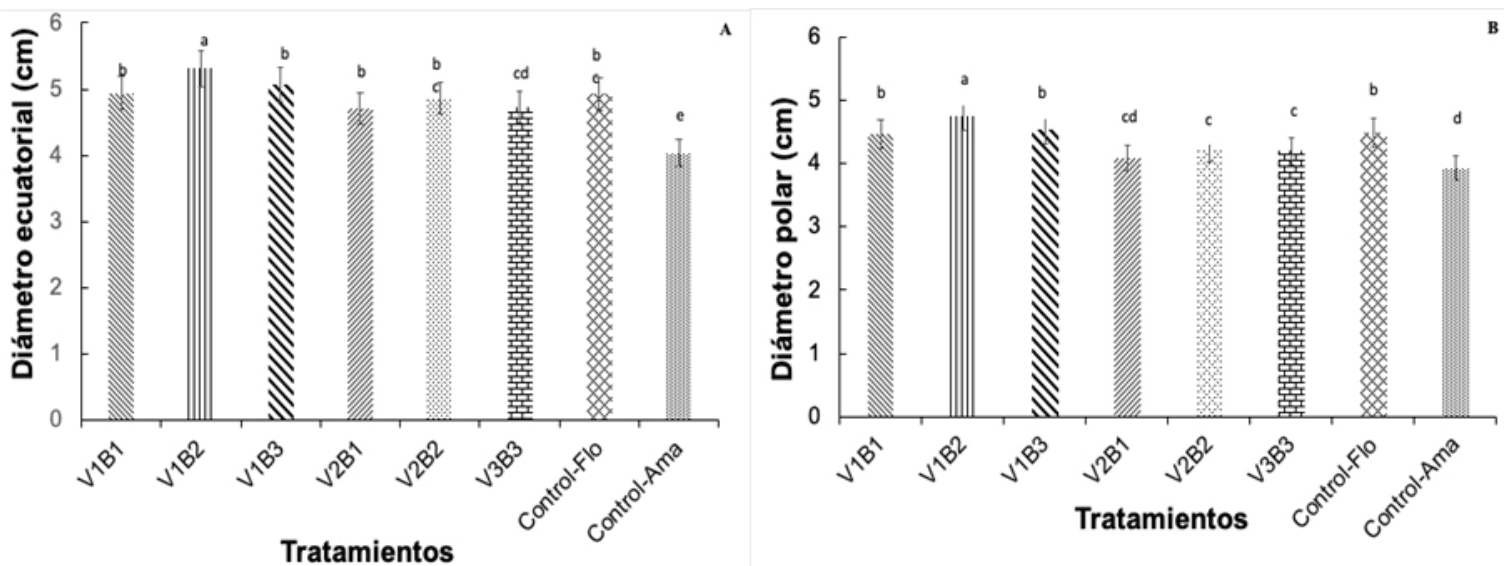

Figura 3. Diámetro ecuatorial (A), diámetro polar (B) del fruto de las variedades Floradade y Amalia mediante la aplicación de tres dosis de quitosano. Las barras en las columnas indican error estándar ( $\pm \mathrm{ES})$; diferentes letras indican diferencias significativas entre los tratamientos (Tukey $\mathrm{p} \leq 0.05$ ). $\mathrm{V}_{1} \mathrm{~B}_{1}=$ Floradade $1 \mathrm{~g} \mathrm{~L}^{-1}, \mathrm{~V}_{1} \mathrm{~B}_{2}=$ Floradade $2 \mathrm{~g} \mathrm{~L}^{-1}, \mathrm{~V}_{1} \mathrm{~B}_{3}=$ Floradade $3 \mathrm{~g} \mathrm{~L}^{-1}, \mathrm{~V}_{2} \mathrm{~B}_{1}=$ Amalia $1 \mathrm{~g} \mathrm{~L}^{-1}, \mathrm{~V}_{2} \mathrm{~B}_{2}=\mathrm{Amalia}$ $2 \mathrm{~g} \mathrm{~L}^{-1}, \mathrm{~V}_{3} \mathrm{~B}_{3}=$ Amalia $3 \mathrm{~g} \mathrm{~L}^{-1}$, control-flo (Floradade agua destilada), control-ama (Amalia agua destilada).

Figure 3. Equatorial diameter (A), polar diameter (B) of the fruit from Floradade and Amalia varieties, by applying three doses of chitosan. Error bars indicate $\pm \mathrm{ES}$; different letters indicate significant differences between treatments (Tukey $\mathrm{p} \leq 0.05$ ). $\mathrm{V}_{1} \mathrm{~B}_{1}=\mathrm{Florada}-$ de $1 \mathrm{~g} \mathrm{~L}^{-1}, \mathrm{~V}_{1} \mathrm{~B}_{2}=$ Floradade $2 \mathrm{~g} \mathrm{~L}^{-1}, \mathrm{~V}_{1} \mathrm{~B}_{3}=$ Floradade $3 \mathrm{~g} \mathrm{~L}^{-1}, \mathrm{~V}_{2} \mathrm{~B}_{1}=$ Amalia $1 \mathrm{~g} \mathrm{~L}^{-1}, \mathrm{~V}_{2} \mathrm{~B}_{2}=$ Amalia $2 \mathrm{~g} \mathrm{~L}^{-1}, \mathrm{~V}_{3} \mathrm{~B}_{3}=$ Amalia $3 \mathrm{~g} \mathrm{~L}^{-1}$, control-flo (Floradade distilled water), control-ama (Amalia distilled water). 

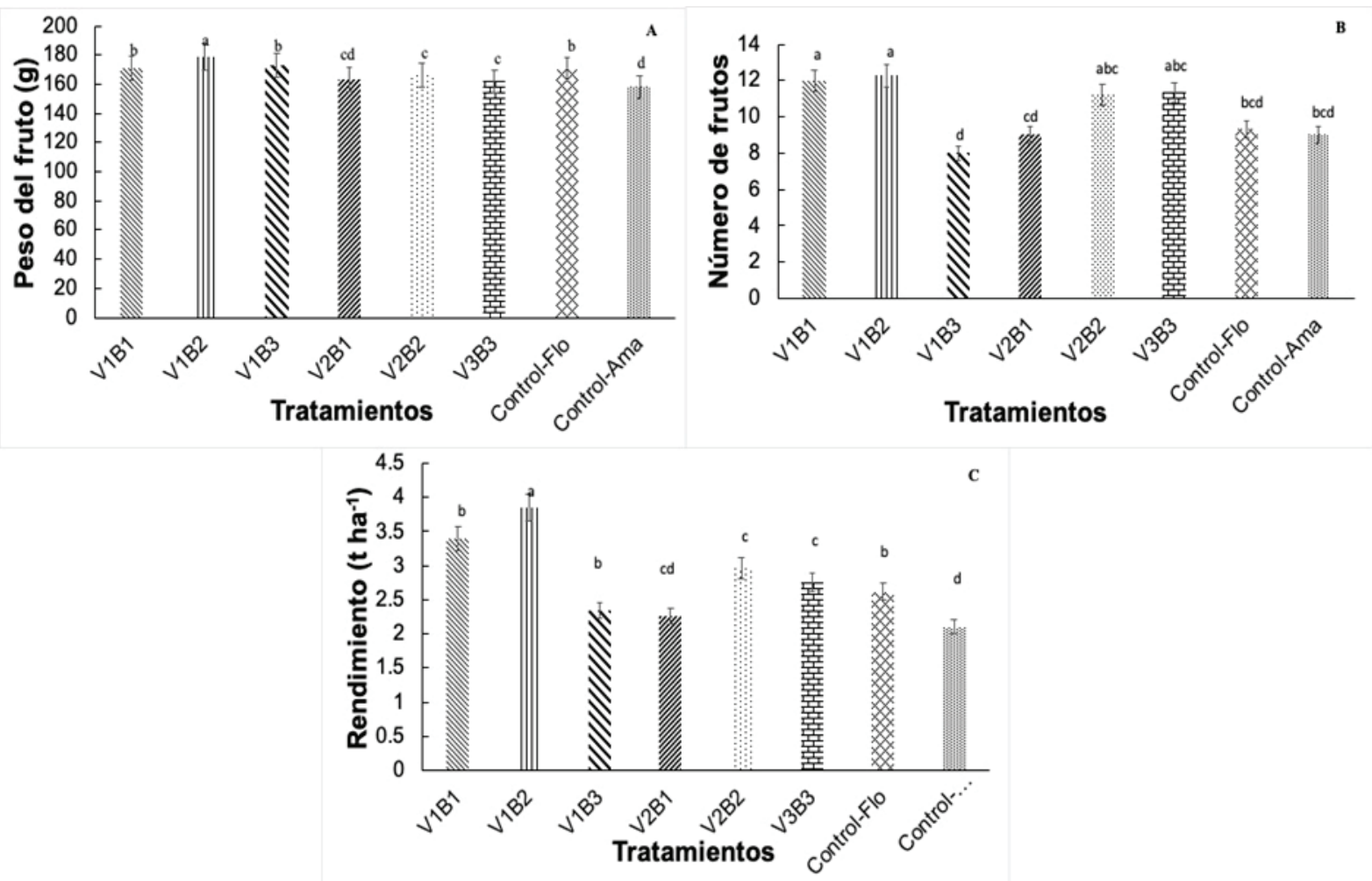

Figura 4. Número de frutos (A), peso del fruto (B) y rendimiento de las variedades Floradade y Amalia mediante la aplicacion de tres dosis de quitosano. Las barras en las columnas indican error estándar ( $\pm \mathrm{ES})$; diferentes letras indican diferencias significativas entre los tratamientos (Tukey $\mathrm{p} \leq 0.05$ ). $\mathrm{V}_{1} \mathrm{~B}_{1}=$ Floradade $1 \mathrm{~g} \mathrm{~L}^{-1}, \mathrm{~V}_{1} \mathrm{~B}_{2}=$ Floradade $2 \mathrm{~g} \mathrm{~L}^{-1}, \mathrm{~V}_{1} \mathrm{~B}_{3}=$ Floradade $3 \mathrm{~g} \mathrm{~L}^{-1}, \mathrm{~V}_{2} \mathrm{~B}_{1}=$ Amalia $1 \mathrm{~g} \mathrm{~L}^{-1}, \mathrm{~V}_{2} \mathrm{~B}_{2}=$ Amalia $_{2}$ $\mathrm{g} \mathrm{L}^{-1}, \mathrm{~V}_{3} \mathrm{~B}_{3}=$ Amalia $3 \mathrm{~g} \mathrm{~L}^{-1}$, control-flo (Floradade agua destilada), control-ama (Amalia agua destilada).

Figure 4. Number of fruits (A), fruit weight $(B)$ and yield of the Floradade and Amalia varieties by applying three doses of chitosan. Error bars indicate $\pm \mathrm{ES}$; different letters indicate significant differences between treatments (Tukey $\mathrm{p} \leq 0.05$ ). $\mathrm{V}_{1} \mathrm{~B}_{1}=$ Floradade $1 \mathrm{~g} \mathrm{~L}^{-1}, \mathrm{~V}_{1} \mathrm{~B}_{2}=$ Floradade $2 \mathrm{~g} \mathrm{~L}^{-1}, \mathrm{~V}_{1} \mathrm{~B}_{3}=$ Floradade $3 \mathrm{~g} \mathrm{~L}^{-1}, \mathrm{~V}_{2} \mathrm{~B}_{1}=$ Amalia $1 \mathrm{~g} \mathrm{~L}^{-1}, \mathrm{~V}_{2} \mathrm{~B}_{2}=$ Amalia $2 \mathrm{~g} \mathrm{~L}^{-1}, \mathrm{~V}_{3} \mathrm{~B}_{3}=$ Amalia $3 \mathrm{~g} \mathrm{~L}^{-1}$, control-flo (Floradade distilled water), control-ama (Amalia distilled water).

rendimiento en la variedad Floradade alcanzando el mayor rendimiento con promedio de $3.85 \mathrm{t} \mathrm{ha}^{-1}$ seguido de la aplicación de $1 \mathrm{~g} \mathrm{~L}^{-1}$ en la misma variedad que presentó un rendimiento de $3.4 \mathrm{t} \mathrm{ha}^{-1}$. Mientras que la dosis de $3 \mathrm{~g} \mathrm{~L}^{-1}$ redujo significativamente el rendimiento en la variedad Floradade presentando un rendimiento de $2.34 \mathrm{t} \mathrm{ha}^{-1}$. En cuanto al tratamiento de menor rendimiento, se registró en el control con la aplicación de agua en la variedad Amalia.

Por lo que el uso de quitosano como bioestimulante tiende a mejorar los rendimientos en los cultivos (Pichyangkura y Chadchawan, 2015), resultados similares a los obtenidos en nuestra investigación, donde la variedad Floradade obtuvo los mayores rendimientos. En este mismo sentido Saavedra et al. (2016) establece que la aplicación de quitosano, no solo mejora el peso de los frutos, el rendimiento en cultivos hortícolas, sino que, también reduce el deterioro poscosecha por enfermedades. Por su parte Rendina et al. (2019) establecen que al aplicar quitosano, este presenta efectos positivos en pepino en las variables de crecimiento y desarrollo. En este mismo sentido, Jiménez et al. (2015) reportan que en concentraciones similares lograban los mayores valores de diámetro ecuatorial en el cultivo del tomate en el cultivar $\mathrm{H}-3108$ y en condiciones de casas de cultivo protegido. Asimismo Reyes et al. (2020) mencionan que concentraciones de $300 \mathrm{mg} \mathrm{L}^{-1}$ mejoraron los rendimientos en tomate. Estos rendimientos de cosecha superiores al del tratamiento sin quitosano, eran de esperarse de acuerdo al seguimiento desde la etapa de germinación hasta la etapa de crecimiento vegetativo. Los bioestimulantes como el quitosano contienen principios activos, que actúan sobre la fisiología de las plantas aumentando su desarrollo y mejorando su productividad en la calidad del fruto, contribuyendo a mejorar la resistencia de las especies vegetales y su rendimiento (González et al., 2012; Rivero et al., 2009).

\section{CONCLUSIONES}

La aplicación de quitosano en dosis de $2 \mathrm{~g} \mathrm{~L}^{-1}$ en tomate de la variedad Floradade presentaron los más altos promedios de germinación, tasa de emergencia y emergencia, desarrollo de radicular, altura de planta, biomasa fresca, diámetro ecuatorial, diámetro polar y rendimiento. La aplicación de quitosano en tomate de la variedad Amalia, indujo a valores mayores de germinación, tasa de emergencia y emergencia, altura de planta a los 45 días, mayor número de frutos. Pero menor peso de frutos y menor rendimiento que la variedad Floradade. 


\section{AGRADECIMIENTOS}

A la Universidad Técnica Estatal de Quevedo, por el apoyo otorgado a través del Fondo Competitivo de Investigación Científica y Tecnológica (FOCICYT) $6^{\text {ta }}$ Convocatoria, a través del proyecto PFOC 6-20-2018 "Evaluación de derivados de Quitosano en la producción sostenible de hortalizas en sistema de cultivo orgánico".

\section{LITERATURA CITADA}

Beier S., y Bertilsson S. 2013. Bacterial chitin degradation mechanisms and ecophysiological strategies. Frontiers in Microbiol. 4 (149):1-12.

Berumen, V. G., Coronado, P., V. A. Ochoa, J. M. A., Chacón, L. P., Gutiérrez, M. 2015. Efecto del quitosano en la inducción de resistencia contra Colletotrichum sp. en mango (Mangifera indica L.) Cv. Tommy Atkins. Investigación y Ciencia. 23(66):16-21.

Brown, P., y Saa, S. 2015. Biostimulants in agriculture. Mini-Review. Front. Plant Sci. 6(671):1-3. doi:10.3389/fpls.2015.00671.

Caro, L. F. J., López, M. L. M., Lizardi, M. J., Argüelles, M. W., Goycoolea, V. F. M., Carvajal, M. E., López, F.Y.L. 2019. Métodos de preparación de nanopartículas de quitosano: una revisión. Biotecnia 21 (3):13-25. https://doi.org/10.18633/ biotecnia.v21i3.1007.

Cestoni, F., De Jovel, G., Urquilla, A. 2006. Perfil de negocios de tomate cherry o cereza hacia el mercado de los Estados Unidos. 73 p. El Salvador. Obtenido de http://www.academia. edu/7215115/perfil de negocios del tomate cherry o cereza hacia el mercado de los Estados Unidos.

Coronel, J. 2009. Alternativas de mejora en el manejo postcosecha de tomate riñon cultivados de Santa Elena. Disertación de Master. Escuela Superior Politecnica del Litoral, Guayaquil, Ecuador.

Devine G. J., Eza D., Ogusuku E., Furlong M. J. 2008. Uso de insecticidas: contexto y consecuencias ecológicas. Revista Peruana de Medicina Experimental y Salud Pública. 25(1): 74-100.

Falcón, R.A., Costales, M.D., González, P.F., Nápoles, G.M. C. 2015. Nuevos productos naturales para la agricultura: las oligosacarinas. Cultivos Tropicales. 36 (1):111-129.

Feicán, M.C., Encalada, A. C.R., Becerril, R. A.E. 2016. Descripción agronómica del cultivo de tomate de árbol (Solanum betaceum Cav.). Agroproductividad. 9(8):78-86.

González, P. D., Costales, D., y Falcón, A. B. 2014. Influencia de un polímero de quitosana en el crecimiento y la actividad de enzimas defensivas en tomate (Solanum lycopersicum L.). Cultivos Tropicales. 35(1):35-42.

González, L. G., Paz, I., Martínez, B., Jiménez, M. C., Torres, J. A. y Falcón, A. 2017. Respuesta agronómica del cultivo del tomate (Solanum lycopersicum, L) var. HA 3019 a la aplicación de quitosana. UTCIENCIA. 2(2):55-60.

Jiménez, A. M. C.; Terrero, S. J. C.; González, G. L. G.; Paz, M. I. y Falcón, R. A. 2015. Evaluación de la aplicación de quitosana sobre parámetros agronómicos del cultivo de tomate H-3108 (Solanum lycopersicum L.) en casas de cultivos protegidos. Centro Agrícola. 42(3):81-88.

González, L., Falcan, A., Jiménez, M. C., Jiménez, L., Silvente, J. y Terrero, J. C. 2012. Evaluación de tres dosis de quitosana en el cultivo de pepino en un periodo tardío Revista amazónica: Ciencia y tecnología. 1(29):134-139.
Iriti, M. y F. Faoro. 2009. Chitosan antitranspirat activity is due to abscisic acid dependent stomatal closure. Env. Exp. Bot. 66(3):493-500.

Kumeta, Y., Inami, Y., Ishimaru, K., Yamazaki, Y., Sameshima, S. R. y Saito, A. 2018. Thermogravimetric evaluation of chitin degradation in soil: implication for the enhancement of ammonification of native organic nitrogen by chitin addition. Soil Science and Plant Nutrition. 64(4): 512-519. doi.org/10.1080/00380768.2018.1457408.

Ministerio de Agricultura, Ganadería, Acuacultura y Pesca (MAGAP). 2014. Cultivo de tomate: Manejo de plagas y enfermedades. 8-14.

Monzón, C.A. 2016. Evaluación del rendimiento del tomate de crecimiento indeterminado (Lycopersicum esculentum Mill) de variedades hibridos utilizando abonos fermentados de gallinaza y cuyaza-abancay. Disertación de licenciatura, Universidad Tecnológica de los Andes, Abancay-Apurímac, Peru.

Morocho, M.T. y Leiva, M.M. (2019). Microorganismos eficientes, propiedades funcionales y aplicaciones agrícolas. Centro Agrícola. 46(2):93-103.

Múnera, C. B. E. 2015. Aplicación de $\mathrm{TiO}_{2}$ modificado con polioxometalatos en la degradación de soluciones acuosas de carbofuran bajo radiación solar simulada, y evaluación del potencial de acople con un sistema biológico. Disertación Doctoral. Universidad del Valle, Santiago de Chile.

Pichyangkura, R. y Chadchawan, S. 2015. Biostimulant activity of chitosan in horticulture. Scientia Horticulturae. 196: 49-65. doi:10.1016/j.scienta.2015.09.031.

Ramírez, A., T. Rodríguez, L. A. y C. Peniche. 2010. Chitin and its derivatives as biopolymers with potential agricultural applications. Biotecnología Aplicada 27(4):270-276.

Reyes, P. J., Enriquez, A. E., Ramírez, A. M., Zuñiga, V. E., Lara, C. y Hernandez, M.L.G. 2020. Efecto del quitosano sobre variables del crecimiento, rendimiento y contenido nutricional del tomate. Revista Mexicana de Ciencias Agrícolas. 11(3):457465.

Reyes, P. J., Ramírez, A.M. A., Rodríguez, P. A. T., Lara, C. L. y Hernández, M. L. G. 2019. Effect of quitomax ${ }^{\circledR}$ on the indicators of growth, phenology and yield of cowpea (Vigna unguiculata L.). Biotecnia 21(1):109-112. https://doi. org/10.18633/biotecnia.v21i1.820.

Rendina, N., Nuzzaci, M., Scopa, A., Cuypers, A. y Sofo, A. 2019. Chitosan-elicited defense responses in Cucumber mosaic virus (CMV)-infected tomato plants. Journal of Plant Physiology. 234(235):9-17. https://doi.org/10.1016/j. jplph.2019.01.003.

Rivero, D., Cruz A., Martínez, B., Ramírez, M. y Rodríguez, A. 2009. Actividad antifúngica in vitro de la quitosana sigma frente a hongos fitopatógenos causantes del manchado del grano en el cultivo de arroz (Oriza sativa). Fitosanidad. 13(2):101107.

Rodríguez, P.A.T., Ramírez, A. M. A., Falcón, R. A., Bautista, B., S. Ventura, Z. E. y Valle, F. Y. 2017. Efecto del Quitomax en el rendimiento y sus componentes del cultivar de arroz (Oryza sativa L.) var. INCA LP 5. Cultivos Tropicales. 38(4):156-159.

Saavedra, G.M., Figueroa, N.E., Poblete, L.A., Cherian, S. y Figueroa, C. R. 2016. Effects of preharvest applications of methyl jasmonate and chitosan on postharvest decay, quality and chemical attributes of Fragaria chiloensis fruit. Food Chem. 1(190):448-453. https://doi.org/10.101.6/j. foodchem.2015.05.107 
Salachna, P. y Zawadzińska, A. 2014. Effect of chitosan on plant growth, flowering and corms yield of potted freesia. Journal of Ecological Engineering. 15(3):97-102. doi:10.12911/22998993.1110223.

SIPA. 2018. Sistema de Informacion Pública Agropecuaria. Disponible en http://sipa.agricultura.gob.ec/index.php/ cifras-agroproductivas.

Terrya, A. E., Falcón, R. A., Ruiz, P. J., Carrillo, S.Y., y Morales, M. H. 2017. Respuesta agronómica del cultivo de tomate al bioproducto QuitoMax ${ }^{\circledast}$. Cultivos Tropicales. 38(1):147-154.
Torres, R. J., Reyes, P.J., González, G. L., Jiménez, P. M., Boicet, F. T., Enríquez, A. E. A., Rodríguez, P. A., Ramírez, A. M.y González, R. J.C. 2018. Respuesta agronómica de dos variedades de maíz blanco (Zeas mays, L.) a la aplicación de quitomax, azofert y ecomic. Biotecnia 20 (1): 3-7. https://doi.org/10.18633/ biotecnia.v20i1.522

Velásquez, C. L. 2008. Algunas posibilidades de la quitina y el quitosano para usos relacionados con la agricultura en Latinoamerica. Revista UDO Agrícola 8(1):1-22. 